\title{
CARACTERIZAÇÃO SAZONAL E INTERANUAL DA PROFUNDIDADE ÓTICA DE AEROSSÓIS EM REGIÃO DE TRANSIÇÃO PANTANAL-CERRADO NO ESTADO DE MATO GROSSO, BRASIL
}

\section{SEASONAL AND INTERANNUAL CHARACTERIZATION OF OPTICAL DEPTH OF AEROSOLS IN PANTANAL CERRADO TRANSITION REGION IN THE STATE OF MATO GROSSO, BRAZIL}

\section{Rafael da Silva Palácios*; Fernando da Silva Sallo; José de Souza Nogueira}

\author{
Universidade Federal de Mato Grosso
}

*Autor para correspondência. E-mail: rafael.pgfa@gmail.com

\begin{abstract}
RESUMO
Estudos relacionados às propriedades óticas de aerossóis atmosféricos são de grande relevância para a pesquisa. Desta forma, o presente trabalho propõe uma caracterização sazonal e interanual da Profundidade Ótica de Aerossóis (AOD) na faixa de $500 \mathrm{~nm}$ para uma região de transição Pantanalcerrado (Características de Cerrado), na região centro sul no estado de Mato Grosso. Os dados de AOD $500 \mathrm{~nm}$ foram adquiridos junto à rede AERONET de 2001 a 2013. Seus valores diários apresentam claramente um comportamento sazonal com mínimos nas estações chuvosas e máximos na estação seca. Os primeiros ficaram concentrados em aproximadamente 0,2 , enquanto que os máximos atingiram valores próximos a 3,0. Com relação à caracterização interanual, percebeu-se que não há comportamento periódico, havendo grandes variações de ano para ano. As médias mensais de AOD $500 \mathrm{~nm}$ foram correlacionadas com os registros mensais de focos de queimada para o estado de Mato Grosso, no entanto, não houve correlação estatisticamente significativa, o que evidencia que tal relação existe, mas que não é direta.
\end{abstract}

Palavras - chave: AERONET. Queima de Biomassa. Depressão Cuiabana.

\begin{abstract}
Studies related to the optical properties of atmospheric aerosols are of great relevance for research. Thus , this paper proposes a seasonal and inter- depth characterization of Aerosol Optical Depth (AOD) in the range of $500 \mathrm{~nm}$ for a Pantanal - Cerrado transition region (Characteristics of Cerrado), in the central south region in the state of Mato Grosso. The AOD $500 \mathrm{~nm}$ data were acquired from AERONET network from 2001 to 2013. The AOD $500 \mathrm{~nm}$ daily values present a seasonal pattern with minimum and maximum in the rainy and dry seasons, respectively. The minimum values were concentrated approximately at 0.2 whereas the maximum values close to 3.0. Regarding the inter-annual characterization, it was noticed that not a periodic behavior, with large variations from year to year. The monthly averages of AOD $500 \mathrm{~nm}$ were correlated with the
\end{abstract}


monthly records of outbreaks of fire for the State of Mato Grosso, nevertheless there was no statistically significant correlation, which shows that such a relationship exists but it is not straightforward.

Keywords: AERONET. Biomass Burning. Cuiabana Depression.

\section{INTRODUÇÃO}

A região centro sul do estado de Mato Grosso é uma região estratégica para análise do perfil ótico dos aerossóis atmosféricos provenientes de queima de biomassa, uma vez que essa região é acometida todos os anos por inúmeros focos de queimadas na estação seca (PALÁCIOS et al., 2014). Tendo em vista que aerossóis atmosféricos são partículas sólidas ou líquidas em suspensão na atmosfera (SEINFELD e PANDIS, 1998), em estudos das variações de propriedades óticas citaram que esses podem ser realizados com análise da Profundidade de Ótica do Aerossol, (AOD) (Aerosol Optical Depth). Este é um indicador da quantidade de aerossóis na coluna vertical da atmosfera (BALAKRISHNAIAH et al., 2011; ZHANG et al., 2014).

O estado de Mato Grosso contém três biomas brasileiros, Amazônia, Cerrado e Pantanal, sendo escolhida uma região de estudo na área de transição entre Pantanal e Cerrado (RODRIGUES et al., 2014). O Cerrado brasileiro, de forma geral, é responsável por grandes emissões de aerossóis devido à queima de biomassa. A extensão desse bioma abrange aproximadamente $2.000 \mathrm{Km}^{2}$, de onde aproximadamente 50\% desse território são considerados terra potencialmente arável (BATLEBAYER et al., 2010). O grande potencial como emissor de material particulado para a atmosfera se deve aos efeitos de queimadas naturais devido à sazonalidade e consequentemente o grande intervalo de tempo em exposição à seca. Esse período se inicia, geralmente, em julho e é consistente de ano para ano, geralmente variando apenas por algumas semanas. Os agravantes, no entanto, estão concentrados entre os meses de agosto e novembro, com algumas variações regionais (SCHAFER et al., 2008).

A AERONET (Aerosol Robotic Network) é uma rede de monitoramento de aerossóis, terrestre de sensoriamento remoto distribuída por todo o globo. Dentre as diversas informações fornecidas por essa rede se encontram os valores de AOD, fornecidos para comprimentos de ondas específicos, entre os quais se encontra o de $500 \mathrm{~nm}$ (HOBEN et al., 2001). Os dados de AOD 500 nm para a região Centro-Oeste do Brasil tanto possibilitam uma caracterização das propriedades óticas da atmosfera local, como auxiliam no entendimento das trocas radiativas na faixa da luz visível entre a atmosfera e a superfície (PALÁCIOS et al., 2014).

As propriedades óticas dos aerossóis também são amplamente estudadas por meio de dados orbitais, dentre tais métodos se destaca a aplicação do sensor MODIS (Moderate Resolution Imaging Spectroradiometer) presente a bordo dos satélites Terra e Agua. Este foi lançado através do programa EOS (Earth Observing System) da NASA para obter medidas remotas diárias de aerossóis sobre a terra e o oceano, e para melhor compreender as quantificações de aerossóis atmosféricos (REMER et al., 2005; WANG et al., 2010).

A caracterização sazonal dos valores de AOD $500 \mathrm{~nm}$ para a região de estudo está intimamente relacionada aos registros de focos de queimadas (PALÁCIOS et al., 2014). Desta forma, uma quantificação desta variável se torna necessária para o maior entendimento dos efeitos provocados pelos aerossóis nos fluxos radiativos ao longo do ano. Nesse sentido o objetivo desse trabalho foi caracterizar sazonalmente a AOD $500 \mathrm{~nm}$ em uma região de transição Pantanal-cerrado no estado de Mato Grosso, a partir de dados adquiridos junto à rede AERONET, levando em consideração o intervalo temporal de 2001 a 2013. 


\section{MATERIAL E MÉTODOS}

A área de estudo se encontra em uma região rural (Fazenda Miranda), localizada a aproximadamente $20 \mathrm{~km}$ da região urbanizada da capital Cuiabá, no estado de Mato Grosso (Figura 1), cujas coordenadas são $16^{\circ} \mathrm{S}$ e $56^{\circ} \mathrm{W}$ a $175 \mathrm{~m}$ de altitude. No local se encontra o fotômetro CIMEL controlado pela rede AERONET. Todos os detalhes sobre a instrumentação, protocolos de medidas, precisão fotométrica, calibração e métodos de processamento podem ser obtidos nos estudos de Holben et al. (1998).

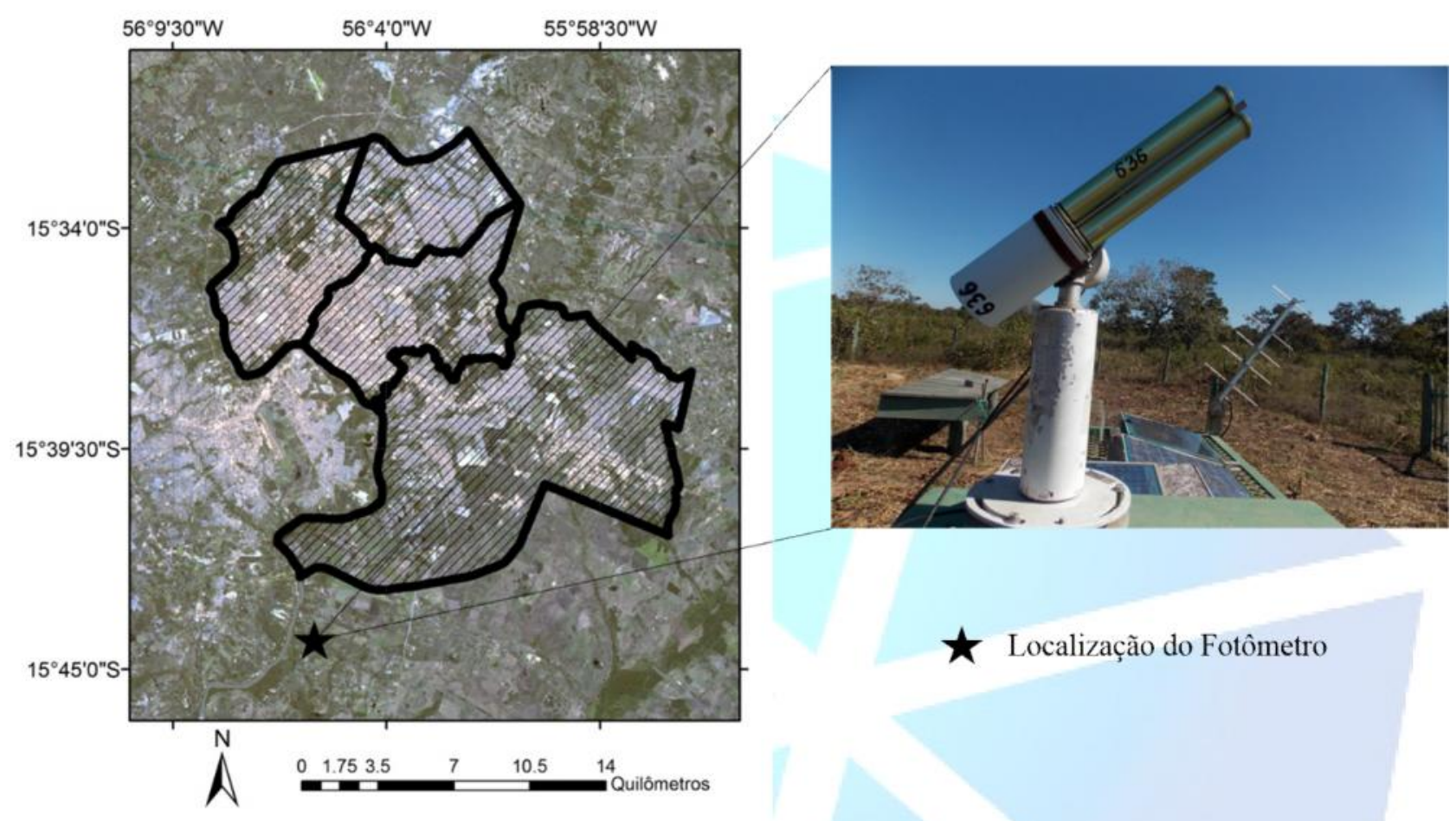

Figura 1. Localização do Fotômetro CIMEL com relação à região urbanizada de Cuiabá.

No presente estudo foram utilizados os dados de AOD $500 \mathrm{~nm}$ de nível 2,0, uma vez que os arquivos de dados da rede AERONET são divididos em três níveis de qualidade: nível 1.0 para os dados brutos, nível 1,5 para dados sem a contaminação de nuvens e nível 2,0 cuja qualidade é certificada pela rede (HOLBEN et al., 1998; SMIRNOV et al., 2000). Foram analisados os dados correspondentes ao período que foi de 2001 a 2013. Os dados utilizados neste trabalho podem ser acessados através do site http://www.inmet.gov.br/portal/index.php?r=bdmep/bdmep. Também foram utilizados neste estudo os dados do Instituto Nacional de Pesquisas Espaciais (INPE), referente ao número de focos de queimadas e incêndios no Estado de Mato Grosso, dados mensais para o período de estudo (2001 a 2013), esses dados estão disponíveis em http://www.inpe.br/queimadas.

No tratamento estatístico dos dados foram analisados os valores máximos e mínimos para cada ano do período de estudo, assim como foram realizadas médias mensais para toda a distribuição de dados. Os valores obtidos nas médias mensais ainda foram correlacionados com os focos de incêndio registrados no estado de Mato Grosso para o mesmo período. Para a determinação da correlação foi estimado o coeficiente de correlação de Spearman, uma vez que a distribuição de dados corresponde a uma distribuição não linear e não homogênea. 


\section{RESULTADOS E DISCUSSÃO}

A distribuição dos valores de AOD $500 \mathrm{~nm}$ apresenta claramente um padrão sazonal, os valores mínimos são registrados no período que vai de dezembro a maio de cada ano, já os máximos estão concentrados de julho a novembro para os respectivos anos. A Figura 2a representa a distribuição diária dos valores de AOD 500 nm, já a Figura 2b evidencia o comportamento sazonal destes, sobrepondo todos os anos por meio da distribuição dos valores em função do dia do ano.
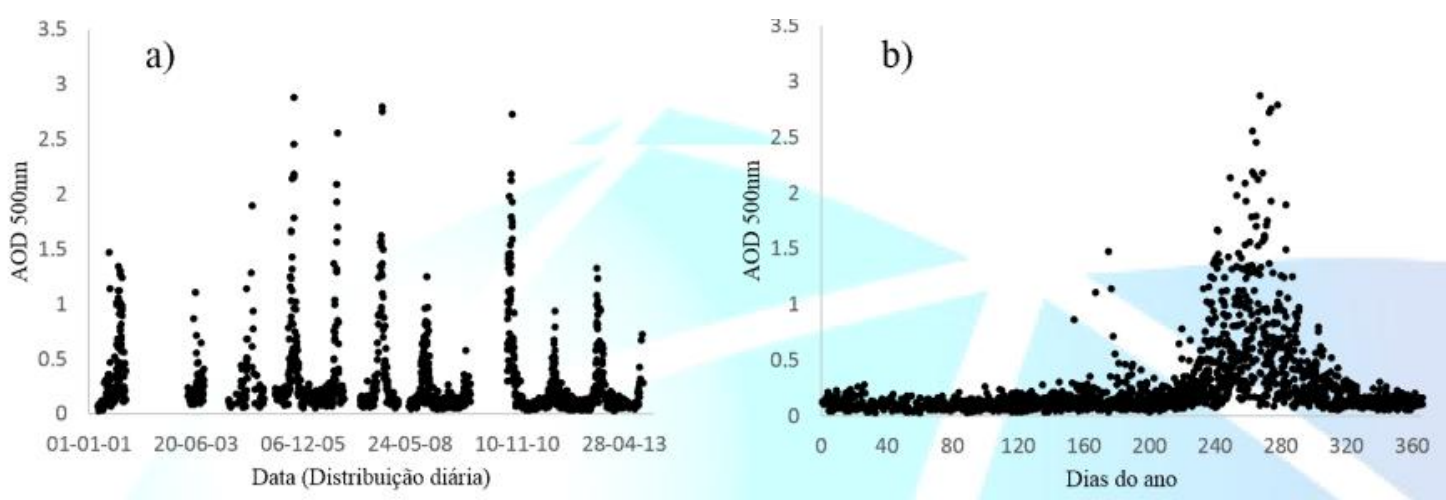

Figura 2. a) Distribuição diária dos valores de AOD 500 nm de 2001 a 2013, b) distribuição dos valores de AOD $500 \mathrm{~nm}$ ao longo do dia do ano.

Observa-se na Figura $2^{\mathrm{a}}$ uma falha consideravelmente longa no ano de 2002, isso devido aos problemas técnicos de calibração do fotômetro. Os valores analisados, no entanto, representam de forma satisfatória os máximos e mínimos de cada ano. Para o ano de 2001 os picos diários de AOD $500 \mathrm{~nm}$ atingiram 1,5 no mês de setembro, em 2003 os registros máximos ficaram aproximadamente em 1,0, no ano de 2004 os valores ultrapassaram 1,0, e em caso isolado atingiu valor bem próximo de 2,0 . Todos os valores foram considerados confiáveis, uma vez que os dados adquiridos junto à rede AERONET foram os de nível 2,0, ou seja, certificado pela rede.

Nos anos de 2005 e 2006 os máximos de AOD $500 \mathrm{~nm}$ atingiram valores próximos a 3,0, enquanto que para o ano de 2007 houve queda desses picos, ficando com apenas alguns valores isolados acima de 2,5. Em 2008 os registros máximos ficaram na faixa de 1,5. Para o período de elevação dos valores de AOD $500 \mathrm{~nm}$ no ano de 2009 houve outra falha na série, que segue até o período de elevação do ano de 2010, onde os picos máximos atingiram valores próximos a 3,0. Já para os anos de 2011 e 2012 os picos ficaram em torno de 1,0 e 1,5, assim como para o ano de 2013.

Os registros de AOD $500 \mathrm{~nm}$ abaixo de 0,5 caracterizam atmosfera praticamente limpa, no entanto, Houghton et al. (1996) afirmaram, que para condições de atmosfera limpa, os valores podem ser inferiores 0,1. O trabalho de Palácios et al. (2014), para os anos de 2010, 2011 e 2012, reporta valores menores que 0,5 para a área de estudo nos períodos de chuva nessa região.

As médias mensais foram analisadas ano a ano conforme mostra a Figura 3. Percebe-se a variação interanual onde o ano de 2010 representou os maiores valores de AOD 500 nm no mês de setembro. 


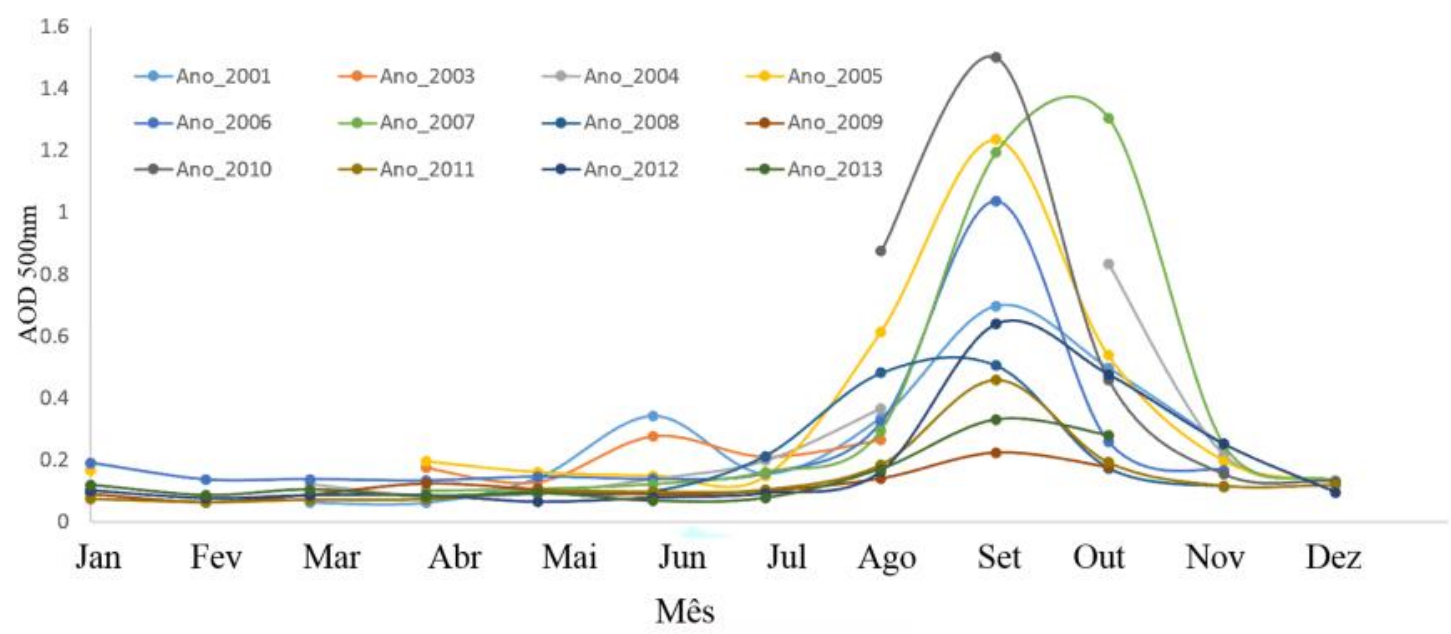

Figura 3. Distribuição dos valores mensais de AOD $500 \mathrm{~nm}$ para os anos de estudo.

Observa-se na Figura 3 que para os meses de dezembro a maio os valores de AOD $500 \mathrm{~nm}$ permaneceram relativamente baixos, entorno de 0,2 , já no mês de junho a uma pequena elevação para os anos de 2001 e 2003. Em todos os anos os valores de AOD $500 \mathrm{~nm}$ começaram a se elevar a partir do mês de julho chegando ao pico máximo no mês de setembro. A de se observar que as amplitudes de ano para ano são variáveis. No ano de 2009 a média mensal para setembro foi relativamente baixa (pouco acima de 0,2), enquanto que em 2010 os picos máximos registrados foram os mais altos de toda a série (acima de 1,4).

Os valores mensais de AOD $500 \mathrm{~nm}$ foram relacionados com os registros mensais de focos de queimada para o estado de Mato Grosso, a Figura 4 representa a distribuição dos valores mensais de AOD $500 \mathrm{~nm}$ e os registros de focos de queimada.

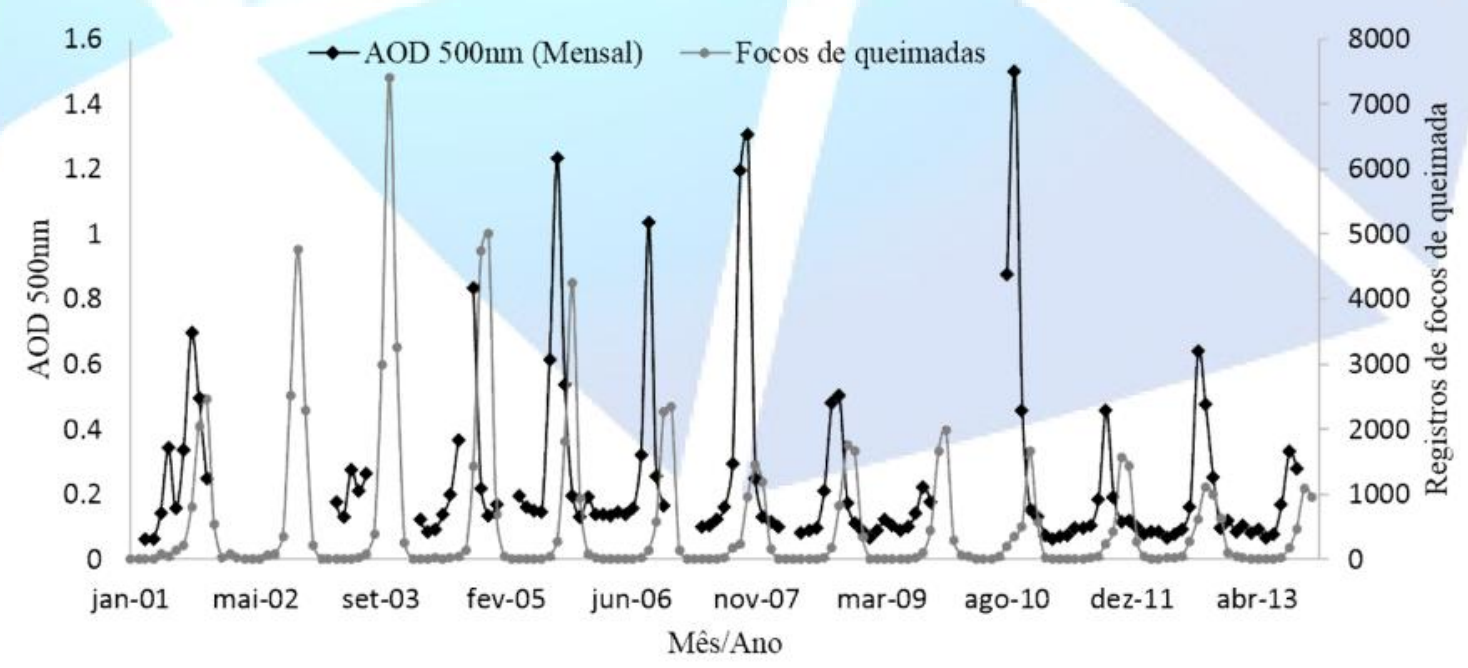

Figura 4. Distribuição mensal dos valores de AOD $500 \mathrm{~nm}$ e registros de focos de queimada no estado de mato Grosso. 
$\mathrm{Na}$ Figura 4 verifica-se que os registros de focos de queimada estão diretamente relacionados com as elevações nos valores de AOD $500 \mathrm{~nm}$, tendo em vista que o aumento dos eventos de queimadas lança para a atmosfera uma grande quantidade de material particulado. Todavia, ha registros de grandes elevações nos valores de AOD $500 \mathrm{~nm}$ que não são acompanhados pelo aumento dos registros de focos de queimada. Para os anos de 2010 e 2011, os registros de focos de queimada foram aproximadamente iguais, já para os valores de AOD $500 \mathrm{~nm} \mathrm{em} 2010$ houve pico máximo relativamente maior do que em 2011.

Calculado o coeficiente de correlação de Spearman para a relação entre AOD $500 \mathrm{~nm}$ e os registros de focos de queimada, não houve uma correlação estatisticamente significativa (0.02), o que evidencia que outras variáveis devem ser consideradas na análise dos valores de AOD 500nm. O trabalho de Palácios et al. (2014) encontrou uma correlação estatisticamente significativa entre as mesmas variáveis, no entanto a série retratada em seu trabalho aborda um intervalo curto da série. Há de se levar em consideração que os registros de focos de queimadas são abordados em nível estadual, sendo que sua contribuição não influenciará necessariamente na área de estudo.

A área de estudo apresenta vegetação característica de Cerrado, o que justificaria em emissões de material por suspensão de poeira devido ao grande tempo de exposição à seca. Outro fator que deve ser levado em consideração é a própria emissão de partículas pela vegetação. De forma geral uma série de variáveis ainda devem ser analisadas a fim de se entender o comportamento sazonal dos valores de AOD 500nm, e as propriedades óticas de forma geral para regiões como a área analisada.

Os valores encontrados para a AOD $500 \mathrm{~nm}$ tanto diário como mensal estão de acordo com a vasta literatura, tal como para a área de estudo retratada por Procópio et al. (2004) e Palácios et al. (2014) como para áreas com mesmas características como o trabalho de Kumar et al. (2013) desenvolvido em região de savana na África.

Através dos resultados obtidos observa-se claramente a variação sazonal dos dados de AOD $500 \mathrm{~nm}$ para a região de estudo. Com relação aos valores máximos e mínimos há de se observar que a metodologia proposta nesse trabalho assume uma análise quantitativa baseada nas variações dos valores de AOD $500 \mathrm{~nm}$ sendo assim possível acompanhar as variações anuais entre os dados, contudo foi possível perceber a grande influência dos focos de queimadas nas variações dos valores de profundidade ótica dos aerossóis.

\section{CONCLUSÃO}

O presente estudo contribui para o entendimento do comportamento das propriedades óticas de aerossóis em regiões com características de Cerrado. Os valores de diários AOD $500 \mathrm{~nm}$ apresentam claramente um comportamento sazonal com mínimos nas estações chuvosas e máximos na estação seca. Os valores mínimos ficaram concentrados em aproximadamente 0.2 enquanto que os máximos atingiram valores próximos a 3.0. Com relação à caracterização interanual, percebeu-se que não a um comportamento periódico, havendo grandes variações de ano para ano. As médias mensais de AOD $500 \mathrm{~nm}$ foram correlacionadas com os registros mensais de focos de queimada para o estado de Mato Grosso, no entanto não houve correlação estatisticamente significativa, o que evidencia que tal relação existe mas que não é direta. A relação entre os valores mensais de AOD $500 \mathrm{~nm}$ e os registros de focos de queimada foi percebida traves das elevações dos valores de AOD $500 \mathrm{~nm}$ quando a um aumento dos valores em registros de focos de queimada. 


\section{AGRADECIMETOS}

Agradecemos a CAPES, pelo apoio financeiro, ao grupo de pesquisa do Programa de Pósgraduação em Física Ambiental e ao Grupo de Estudos do Instituto de Física da USP pela utilização dos dados da rede AERONET.

\section{REFERÊNCIAS BIBLIOGRÁFICAS}

BALAKRISHNAIAH, G., KUMAR, K.R., KUMAR REDDY, B.S.K., GOPAL, K.R., REDDY, R.R., REDDY, L.S.S., AHAMMED, Y.N., NARASIMHULU, K., MOORTHY, K.K., BABU, S.S. Analysis of optical properties of atmospheric aerosols inferred from spectral AODs and Ångström wavelength exponent. Atmospheric Environment, v. 45, p. 1275-1285, 2011.

BATLLE-BAYER, L.; BATJES, N.H; BINDRABAN, P.S. Changes in organic carbon stocks upon land use conversion in the Brazilian Cerrado: A review. Agriculture, Ecosystems and Environment, v. 137, p. 47-58, 2010.

HOLBEN, B.N., ECK, T.F., SLUTSKER, I., TANRÉ, D., BUIS, J.P., SETZER, A., VERMOTE, E., REAGAN, J.A., KAUFMAN, Y.J., NAKAJIMA, T., LAVENU, F., JANKOWIAK, I., SMIRNOV A. AERONET - a federated instrument network and data archive for aerosol characterization. Remote Sensing of the Environment, v. 66, n. 1, p. 1-16, 1998.

HOUGHTON, J. T.; MEIRA FILHO, L. G.; CALLANDER, B. A.; HARRRIS, N.;

KATTENBERG, A.; MASKELL, K. (Eds) Climate Change 1995: The Science of Climate Change. Cambridge University Press, Cambridge, UK, 1996.

KUMAR, K.R., SIVAKUMAR, V., REDDY, R.R., GOPAL, K.R., ADESINA, A.J. Inferring wavelength dependence of AOD and Ångström exponent over a sub-tropical station in South Africa using AERONET data: Influence of meteorology, long-range transport and curvature effect.

Science of the Total Environment, v. 461-462, p. 397-408, 2013.

PALÁCIOS, R.S., SALLO, F.S., do PRADO, M.J., de MUSIS, C.R., NOGUEIRA, J.S. Análise da profundidade ótica de aerossóis e coeficiente de Angstron no Cerrado Mato-grossense. Revista Brasileira de Climatologia, v. 14, p. 173-185, 2014.

PROCOPIO, A.S., ARTAXO, P., KAUFMAN, Y.J., REMER, L.A., SCHAFER, J.S., HOLBEN, B.N. Multiyear analysis of amazonian biomass burning smoke radiative forcing of climate.

Geophysical Research Letters, v. 31, L03108, p. 1-4, 2004.

REMER, L. A., KAUFMAN, Y. J., TANRÉ, D., MATTOO, S., CHU, D. A., MARTINS, J. V., LI, R. R., ICHOKU, C., LEVY, R. C., KLEIDMAN, R. G., ECK, T. F., VERMOTE, E., HOLBEN, B. N. The MODIS Aerosol Algorithm, Products, and Validation. Journal of the Atmospheric Sciences, v. 62, p. 847-973, 2005.

RODRIGUES, T. R.; G. L. VOURLITIS; F. D. A. LOBO; R. G. DE OLIVEIRA; J. D. S. NOGUEIRA. Seasonal variation in energy balance and canopy conductance for a tropical savanna ecosystem of south central Mato Grosso, Brazil, J. Geophys. Res. Biogeosci., v. 119, p. 1-13, 2014. 
SEINFELD, J. H.; PANDIS, S. N.; Atmospheric Chemistry and Physics: From Air Pollution to Climate Change. New York: John Wiley \& Sons, Inc, 1998.

SCHAFER, J.S.; ECK, T.F.; HOLBEN, B.N.; ARTAXO, P.; DUARTE, A. F. Characterization of the optical properties of atmospheric aerosols in Amazônia from long-term AERONET monitoring (1993-1995 and 1999-2006). Journal of Geophysical Research, v.113, D04204, p. 1-16, 2008.

SMIRNOV, A., HOLBEN, B.N., ECK, T.F., DUBOVIK, O., SLUTSKER, I. Cloud screening and quality con trol algorithms for the AERONET data base. Remote Sensing of the Environment, v.73, p. 337-349, 2000.

WANG, L., WANG, Y., XIN, J., LI, Z., WAN, X. Assessment and comparison of three years of Terra and Aqua MODIS Aerosol Optical Depth Retrieval (C005) in Chinese terrestrial regions. Atmospheric Research, v. 97, p. 229-240, 2010.

ZHANG, Z., WENIG, M., ZHOU, W., DIEHL, T., CHAN, K., WANG, L. The contribution of different aerosol sources to the Aerosol Optical Depth in Hong Kong. Atmospheric Environment, v. 83, p. $145-154,2014$. 